\title{
Neonatal Sinus Bradycardia, AE
}

National Cancer Institute

\section{Source}

National Cancer Institute. Neonatal Sinus Bradycardia, AE. NCI Thesaurus. Code

C154933.

An adverse event in a newborn characterized by an abnormally low heart rate for age. 